\title{
Influence of accelerometer signal filtering on automatic detection of gait impact parameters.
}

\author{
A. Camacho-García, R. Llinares, A.G. Lucas-Cuevas, P. Pérez-Soriano
}

\begin{abstract}
Filtering the signal recorded by an accelerometer is essential to remove noise recorded by the sensor, but in order to calculate gait parameters properly, the choice of a suitable cutoff frequency of the filter is critical.

This paper evaluates the influence of the filter cutoff frequency in the calculation of the parameters: vertical peak tibial acceleration and acceleration rate. The accelerometer signal filtering with low-pass filter with cutoff frequency below $50 \mathrm{~Hz}$ gives good results in the calculation of peak tibial acceleration but produces estimations of the acceleration rate below its real value.
\end{abstract}

Keywords-Gait analysis, accelerometry, cutoff frequency, filter, acceleration rate.

\section{Introduction}

Studying the movement of different parts of the body of an athlete in sports is a tool used in biomechanics to improve performance and reduce the injury risk of the athlete[1].

In the field of biomechanics applied to the study of movement, several techniques have been used: Torque platforms, footprint analysis, optical gait analysis and accelerometry among other techniques.

Accelerometers have advantages over other techniques because they are lightweight, low cost, and low power consumption devices that can be used as portable equipment in a free environment. Therefore, they provide real time data from a large number of movements without being restricted to the laboratory environment. They have been used successfully in the study of other types of activities [2].

A common technique is to place several triaxial accelerometers (that allow the registration of 3 different signals corresponding to three mutually orthogonal axes) in different parts of the body and measure relationships between them. The signal analysis provides measures such as cadence, stride length and symmetry of the step. The loads caused by repeated impacts that occur when doing physical exercise have been associated with various adverse effects, such as stress fractures or pain in the lower back [3].

\section{A. Camacho-García, R. Llinares}

Dept. Comunicaciones / Universitat Politècnica de Valencia Spain

A.G. Lucas-Cuevas, P. Pérez-Soriano

GIBD / Universidad de Valencia

Spain
This article evaluates the influence of the cutoff frequency of a low pass filter in the performance of an automatic analysis tool of accelerometer signals recorded in different parts of the body during exercise to provide running stride parameters (stride length, stride rate) [4] and impact acceleration parameters (head and peak tibial acceleration, impact magnitude, acceleration rate, and shock attenuation) [5], [6].

For this study we proceeded as follows:

- Recorded accelerometer signals: two accelerometers were placed on several athletes and data were recorded during running.

- Analysis of events: events were evaluated by an expert in biomechanics to mark points of interest in the signals.

- Software analysis: a software was developed to automatically analyze the events without the support of the expert.

- Effects of the cutoff frequency of the filter: the signals were filtered using different cutoff frequencies and the program performance in the calculation of parameters was evaluated

\section{Record accelerometers signals}

A group of 38 healthy male and female runners aged between 24 and 35 years were involved in the study. Each runner was placed one triaxial accelerometer (Sportmetrics, Spain; sampling frequency $500 \mathrm{~Hz}$, total mass: $2.5 \mathrm{~g}$; dimensions: $40 \mathrm{~mm} \times 22 \mathrm{~mm} \times 12 \mathrm{~mm}$ ) on the tibia and on the head (the vertical axis of the accelerometers were aligned to be parallel to the long axis of the shank). Accelerometer signals were recorded while running on treadmill at a controlled speed of $3.33 \mathrm{~m} / \mathrm{s}$ under various conditions of fatigue and using different types of footwear, in order to get a database that spans multiple conditions to verify the validity of the work.

The accelerometer signals were sampled for 10 seconds at 500 samples per second. The recordings were checked to ensure no abnormal data due to a bad disposition or malfunction of the sensors were produced. The signals were cut to select the most stable signal period and to limit the study to 5 seconds.

The frequency analysis of the recorded signals showed that most of the power was below $100 \mathrm{~Hz}$ and also contained a high noise level in areas close to the Nyquist frequency, so that the captured signals were low-pass filtered using a Chebyshev type II filter of order 8 with $140 \mathrm{~Hz}$ cutoff frequency and ripple in the stopband of $40 \mathrm{~dB}$. 


\section{Analysis of events}

As the vertical axes of the accelerometers are those that provide more information for the detection of points of interest, the vertical component of the accelerometer at the tibia was used as the reference signal to mark points of interest.

An expert in biomechanics studied the signals of the vertical axis of the accelerometer placed on the tibia, separated each step and marked as wrong those steps that were not recorded properly.

In the steps that were recorded successfully, he marked Fig. 1. the maximum acceleration occurred at the moment the foot contacted the ground and the minimum acceleration just before it (used to calculate the acceleration rate).



Fig. 1. Maximum acceleration and minimum acceleration just before it. Vertical tibia acceleration (up). Vertical head acceleration (down). Arbitrary units

\section{Software analysis}

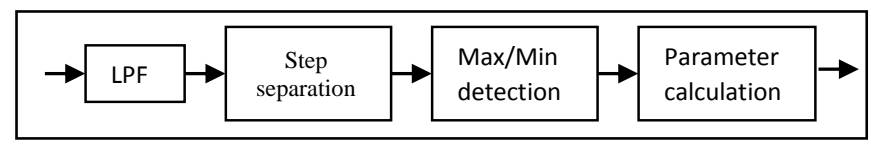

Fig. 2. Step analysis procedure.

The scheme of the implemented software to analyze the signals and perform automatic detection of points of interest is shown in Fig. 2.

\section{A. $\quad \boldsymbol{L P F}$}

The first block consisted of a low-pass filter whose effects we aimed to analyze in this article and whose objective was to eliminate the noise captured by the sensors. Different types of filters were used to evaluate which is the best in the calculation of the studied parameters.

\section{B. Step separation}

In this block, the repetition period of the signal was calculated using the auto-correlation. Then the signal was divided into segments whose size was equal to the period.

As each measurement had a duration of 10 seconds and as the stride duration during running lasts less than one second, each file provided about 10 segments.

\section{Max/Min detection.}

For each signal segment, the local maximum associated with the foot contact with the ground and the local minimum that occurred just before this maximum were calculated.

The foot contact with the ground did not always match the global maximum of the acceleration signal. To locate this point correctly, we used the fact (empirically verified) that this maximum was followed by a very sharp decrease of acceleration to quickly reach a local minimum (less than 50 ms duration).

In addition to previous peaks, we also located the global maximum and minimum of each segment in the tibia and head.

\section{Parameter calculation.}

Based on the above values, we calculated running stride parameters (stride length, stride rate) and impact acceleration parameters (head and tibial peak acceleration, impact magnitude, acceleration rate, and shock attenuation).

In this article, we focused only on the influence of the cutoff frequency of the low pass filter described at point $\mathrm{A}$ in the accuracy to detect the time of ground contact and acceleration rate.

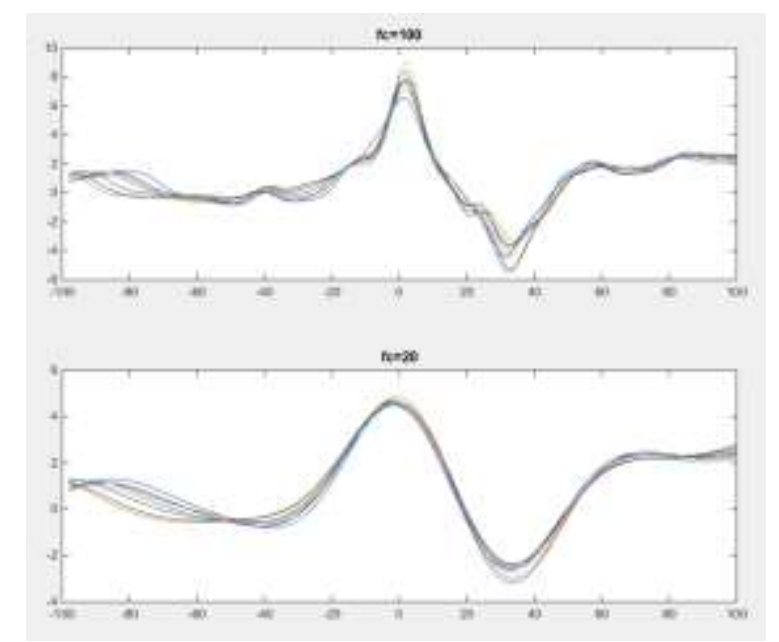

Fig. 3. 8 segments of the accelerometer signal of a single runner centered in ground contact. Low pass $\mathrm{fc}=100 \mathrm{~Hz}$ (up) and $\mathrm{fc}=20 \mathrm{~Hz}$ (down). Time (milliseconds) amplitude (g)

\section{v. Effects of the cutoff frequency of the filter analysis}

To view the effect produced by the LPF block, Fig. 3. shows all segments of the accelerometer signal successfully registered of a single runner. The center of each segment $(0$ $\mathrm{ms}$ ) is the moment when ground contact occurs every stride (maximum automatically detected). The top graph reflects the use of a low pass filter Fig. 1. with $\mathrm{fc}=100 \mathrm{~Hz}$ while the one at the bottom shows the same accelerometer signal but using a filter having $\mathrm{fc}=20 \mathrm{~Hz}$.

Reducing the cutoff frequency of the low pass filter we observed:

- The signal shape is smoothed. 
- The peaks amplitudes are reduced.

- Differences between segments are lower.

Modifications that filters produce in signals depend not only on the cutoff frequency but also on the type of filter used. To evaluate the effect that causes the filter type in the analyzed parameters, in this study Butterworth and Chebyshev Type II of order 2, 4, 6 and 8 were used. In addition, for each of these filters two configurations were used: forward and forward-backward[7].

\section{A. Accuracy detecting the time of ground contact.}

A low-pass Butterworth filter of order 2, 4, 6 and 8, produces an average delay of 11,23, 34 and 44 milliseconds in the detection of the maximum of accelerometry of the signals used in this work. These values are reduced to 1, 2, 3 and 5 milliseconds when the cutoff frequency of the applied filter is $120 \mathrm{~Hz}$. The same effects are observed when using Chebyshev Type II filters:

- The delay increases as the filter order increases.

- The delay decreases as the cutoff frequency increases.

The above effects are observed when a forward filtering is done but not when forward-backward filtering is done. Forward-backward filters have zero-phase and theoretically do not produce shifts of time, therefore offsets measured in the signals we worked with were close to zero (less than 1 millisecond). An exception occurred when using filters with cutoff frequency lower than $40 \mathrm{~Hz}$ : advances greater than 2 milliseconds were measured, probably produced by the influence of the minimum after the maximum in these signals.

As running stride parameters are not dependent on the moment the maximum occurs but the time between one maximum and the next one, this delay will not cause errors in measurements. To evaluate the influence of the used filter in running stride parameters, the error is calculated as the difference between the time when the maximum occurs and the time when it is detected (correcting the delay introduced by the filter).

TABLE I. ERROR IN DETECTION OF GROUND CONTACT (TIME IN MS)

\begin{tabular}{|l|r|c|c|c|c|c|c|}
\hline & \multicolumn{7}{|c|}{ Filter cutoff frequency (Hz) } \\
\hline Filter type & $\mathbf{2 0}$ & $\mathbf{4 0}$ & $\mathbf{6 0}$ & $\mathbf{8 0}$ & $\mathbf{1 0 0}$ & $\mathbf{1 2 0}$ & $\mathbf{1 4 0}$ \\
\hline Forw.Butter.2 & 2,83 & 2,24 & 0,53 & 1,02 & 0,50 & 0,55 & 0,90 \\
\hline Forw.Butter.8 & 1,08 & 2,87 & 2,37 & 2,00 & 0,57 & 0,34 & 0,87 \\
\hline Forw.Cheby.2 & 15,76 & 3,91 & 0,94 & 2,22 & 0,90 & 0,75 & 1,00 \\
\hline Forw.Cheby.8 & 2,84 & 1,03 & 2,24 & 0,70 & 0,97 & 0,67 & 0,45 \\
\hline F/back.Butter.2 & 4,02 & 2,20 & 0,70 & 0,40 & 0,25 & 0,08 & 0,11 \\
\hline F/back.Butter.8 & 2,88 & 2,47 & 0,73 & 0,52 & 0,16 & 0,06 & 0,07 \\
\hline F/back.Cheby.2 & 14,81 & 4,12 & 2,75 & 0,93 & 0,86 & 0,70 & 0,52 \\
\hline F/back.Cheby.8 & 2,17 & 1,09 & 0,78 & 0,57 & 0,37 & 0,06 & 0,07 \\
\hline
\end{tabular}

TABLE I. contains the mean square errors measured when applying different filter types, and it can be drawn the following conclusions:

- Chebyshev filters of order 2 are not suitable because they produce much higher errors than the other filters for all cutoff frequencies.
- Cutoff frequency below $60 \mathrm{~Hz}$. produce significant errors, but above this value there is not dependence of error on frequency.

- Butterworth filters provide better results than Chebyshev.

- Forward-backward filtering provide better results than forward filtering.

\section{B. Accuracy detecting the acceleration of ground contact.}

The correct measurement of the maximum acceleration occurred at the moment the foot contact the ground is very important since it is used in the calculation the impact acceleration parameters.

TABLE II. ERROR IN DETECTION OF MAXIMUM ACCELERATION ( \%)

\begin{tabular}{|l|c|c|r|r|r|r|c|}
\hline & \multicolumn{7}{|c|}{ Filter cutoff frequency (Hz) } \\
\hline Filter type & $\mathbf{2 0}$ & $\mathbf{4 0}$ & \multicolumn{1}{|c|}{$\mathbf{0}$} & $\mathbf{8 0}$ & $\mathbf{1 0 0}$ & $\mathbf{1 2 0}$ & $\mathbf{1 4 0}$ \\
\hline Forw.Butter.2 & 30,93 & 12,51 & 5,44 & 2,59 & 1,16 & 0,47 & 0,24 \\
\hline Forw.Butter.8 & 40,13 & 16,81 & 7,39 & 3,12 & 1,18 & 0,38 & 0,29 \\
\hline Forw.Cheby.2 & 82,55 & 37,27 & 22,53 & 14,86 & 9,11 & 5,32 & 2,97 \\
\hline Forw.Cheby.8 & 44,80 & 19,64 & 9,23 & 3,81 & 1,47 & 0,41 & 0,07 \\
\hline F/back.Butter.2 & 39,91 & 17,95 & 8,60 & 4,15 & 1,93 & 0,84 & 0,33 \\
\hline F/back.Butter.8 & 35,13 & 17,01 & 5,99 & 2,27 & 0,45 & 0,02 & 0,00 \\
\hline F/back.Cheby.2 & 86,61 & 45,40 & 29,36 & 19,55 & 13,27 & 8,20 & 4,64 \\
\hline F/back.Cheby.8 & 37,30 & 18,95 & 6,80 & 2,85 & 0,63 & 0,01 & 0,00 \\
\hline
\end{tabular}

TABLE II. contains the mean errors of this maximum measured applying different filter types, and it can be drawn the following conclusions:

- Chebyshev filters of order 2 are not suitable because they produce much higher errors than the other filters for all cutoff frequencies.

- Error decreases as the filter cutoff frequency increases. Cutoff frequencies above $60 \mathrm{~Hz}$. produce errors less than $10 \%$, and cutoff frequencies above $80 \mathrm{~Hz}$. produce errors less than $5 \%$.

- Butterworth filters provide slightly better results than Chebyshev.

- Forward-backward filtering provide slightly better results than forward filtering.

\section{Accuracy detecting the acceleration rate.}

The acceleration rate [5] measures the slope of the graph of acceleration when plot versus time, and errors in the estimation of time and amplitude of the maximum discussed in the preceding paragraphs strongly affect the accuracy in the calculation of this parameter.

Every point in Fig. 5. represents the mean value of the acceleration rate automatically calculated for all stride of a runner detected correctly. These points are connected with lines so that each line represents the acceleration rate of a single runner calculated using filters with different cutoff frequencies. 


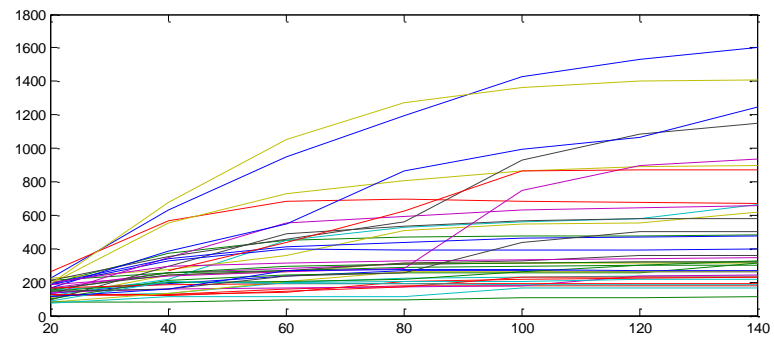

Fig. 4. Acceleration rate $(\mathrm{G} / \mathrm{s})$ calculated for 38 runners as a function of the cutoff frequency $(\mathrm{Hz})$.

All lines show a rising trend which converges asymptotically to a final value (the exact value of the acceleration rate) .When the exact value of the acceleration rate is increased the final value is reached for higher values of the cutoff frequency. To study this effect, Fig. 5. groups all lines of Fig. 5. in two separate lines: the green line is the average of all high acceleration rate (final value is over $500 \mathrm{~g} / \mathrm{s}$ ) lines in Fig. 5. and the blue line is the average of all low acceleration rate (final value is less than $500 \mathrm{~g} / \mathrm{s}$ ) lines in Fig. 5.

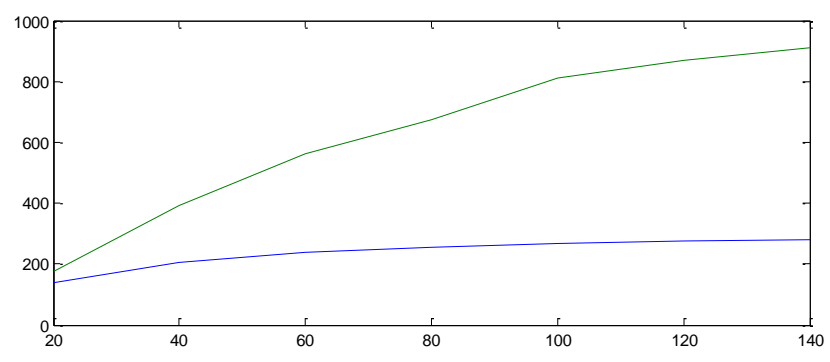

Fig. 5. Acceleration rate (G/s) calculated for two groups of runners as a function of the cutoff frequency $(\mathrm{Hz})$ : green line for final value more than $500 \mathrm{G} / \mathrm{s}$ and blue line for final value less than $500 \mathrm{G} / \mathrm{s}$ ).

Studying the error in the calculation of the slope (difference between the calculated value and the exact value) in 0and Fig. 5. the following facts are observed:

- The error of the calculated acceleration rate decreases as the filter cutoff frequency increases.

- When the exact value of the acceleration rate is high, high cutoff frequency filters are needed: To achieve an error lower than $10 \%$ in the acceleration rate, cutoff frequencies higher than $60 / 100 \mathrm{~Hz}$ are needed for low/high acceleration rates.

The above graphs were carried out using order 2 Butterworth filters. Comparing the results when using other types of filters we realize that:

- Filter order: increasing the filter order, especially using forward-backward filters, abnormalities appear in the trend of the graphs (the error of the calculated acceleration rate do not decreases as the filter cutoff frequency increases).

- Filter type: No significant differences between the results are observed when using Chebyshev or Butterworth filters.

- Forward or forward-backward filters: If a forwardbackward filter is used, higher values of fc well to detect the slope needed. (particularly when the slopes are high).

\section{vi. Conclusions}

This paper proposes a method for automatically detecting the point of contact with the ground in the race, from accelerometer signals to provide running stride parameters and impact acceleration parameters.

We analyzed the effect of the low pass filter on the accuracy of the results and found that the use of filters with cut-off frequencies below $60 \mathrm{~Hz}$ and present problems detecting ground contact time and ground contact acceleration. Moreover Chebyshev filters of order 2 do not work properly with this kind of signals.

Increasing the cutoff frequency of the filter improves the estimation of the acceleration rate properly. To calculate the acceleration rate properly cutoff frequency filters above $100 \mathrm{~Hz}$ may be needed.

Using this model with appropriate parameters more than $90 \%$ of the ground contact points correctly detected.

\section{VII.Acknowledgment}

This work has been sponsored by the Generalitat Valenciana: application 09.02.03.542.50.7 budget line T4015 grant from the "Conselleria de Educación, Cultura y Deporte", aid for conducting R \& D for emerging research groups corresponding to the call set out in Annex IX, the Order 64/2014, of July 31, the "Conselleria de Educación, Cultura y Deporte" (DOCV no. 7.332, of August 5, 2014). Record GV / 2015/067.

\section{References}

[1] A. H. Gruber, K. a. Boyer, T. R. Derrick, and J. Hamill, "Impact shock frequency components and attenuation in rearfoot and forefoot running,” J. Sport Heal. Sci., vol. 3, no. 2, pp. 113-121, 2014.

[2] M. Yang, H. Zheng, H. Wang, S. McClean, and D. Newell, "iGAIT: An interactive accelerometer based gait analysis system," Comput. Methods Programs Biomed., vol. 108, no. 2, pp. 715-723, 2012.

[3] J. Mizrahi, O. Verbitsky, and E. Isakov, "Shock accelerations and attenuation in downhill and level running," Clin. Biomech., vol. 15, no. 1, pp. 15-20, 2000.

[4] N. Chambon, N. Delattre, N. Guéguen, E. Berton, and G. Rao, "Is midsole thickness a key parameter for the running pattern?," Gait Posture, vol. 40, no. 1, pp. 58-63, 2014.

[5] A. M. Duquette and D. M. Andrews, "Comparing methods of quantifying tibial acceleration slope,” J. Appl. Biomech., vol. 26, no. 2, pp. 229-233, 2010.

[6] A. M. Duquette and D. M. Andrews, "Tibialis anterior muscle fatigue leads to changes in tibial axial acceleration after impact when ankle dorsiflexion angles are visually controlled," Hum. Mov. Sci., vol. 29, no. 4, pp. 567-577, 2010.

[7] F. Gustafsson, "Determining the Initial States in Forward-Backward Filtering.” Linköping University, 1994. 